\title{
The Public Good Game on Graphs: Can the Pro-Social Behavior Persist?
}

\author{
Roberto da Silva \\ Instituto de Informática - UFRGS \\ Av. Bento Gonçalves, 9500 - Campus do Vale \\ Bairro Agronomia - Porto Alegre - RS -Brasil \\ CEP: 91501-970 Caixa Postal: 15064 \\ rdasilva@inf.ufrgs.br
}

Received on 10 September, 2007

\begin{abstract}
This communication proposes new alternatives to study the pro-social behavior in artificial society of players in the context of public good game via Monte Carlo simulations. Here, the pro-social aspect is governed by a binary variable called motivation that incites the player to invest in the public good. This variable is updated according to the benefit achieved by the player, which is quantified by a return function. In this manuscript we propose a new return function in comparison with other one explored by the same author in previous contributions. We analyze the game considering different networks studying noise effects on the density of motivation. Estimates of pro-sociability survival probability were obtained as function of randomness $(p)$ in small world networks. We also introduced a new dynamics based on Gibbs Sampling for which the motivation of a player (now a $q$-state variable) is chosen according to the return of its neighbors, discarding the negative returns.
\end{abstract}

Keywords: Public good game; Monte Carlo Simulations; Artificial societies

\section{INTRODUCTION}

The Evolutionary game theory $[1,3]$ has contributed meaningfully for the understanding of the emerging aspects in artificial societies modeled with players rules that evolve along time. This approach has incorporated dynamic aspects to the classical game theory, becoming an interesting laboratory to study economic and social quantities of an artificial society like wealth distribution, pro-social behavior, cooperation and other similar measures that can model some real situations.

Many games have been considered to explain properties originated from bargaining, cooperation, competition and why not altruism, fairness, selfishness. From the experimental economics ${ }^{1}$, an interesting game was proposed to explain the prosocial behavior (the behavior intended to benefit others in the society as a whole), i.e., how altruistic actions can lead to good situations where benefits are distributed for a group of people. This game is known as the public good game $[1,2,7]$. The original version of this game models the public spending for the community, like roads, bridges, libraries. Players are offered the opportunity to invest their money into a common pool. The profits, which are obtained from tolls and membership fees are equally distributed among all participants irrespective of their contributions after the duplication or triplication of the stored amount in the pool to encourage the contribution. However, each player unaware of the contribution of the other players would invest nothing which corresponds to the rational behavior. Thus, for purely rational players the dominant solution is to default, i.e., absence of pro-social behavior!

Several aspects of this game have been studied by economists in experiments with human beings. [4] has conducted

\footnotetext{
${ }^{1}$ http://en.wikipedia.org/wiki/Experimental_economics
}

experiments in United States and Japan aiming to study the voluntary participation in the public goods. The work claims that japanese are more likely to act spitefully which leads to more efficient contributions when compared with American subjects.

Other experimental results from [5] show that in institutions, i.e., group of people interacting according to the public good game are more competitive when punishments among the participants are adopted, i.e., sanctioning institutions are more efficient. Group size effects are also studied in the provision of public good via experiments of voluntary contributions [6] which shows that a group's ability to provide the optimal level of a public good is not inversely proportional to group size as expected previously by premises.

Alternatively, computational and analytical results can also give some understanding of important properties of public goods game. In our previous contributions [7-9], a version of this game with a binary motivation parameter was introduced to lead the investment among the players where the return per agent is calculated according to

$$
\begin{aligned}
R_{i}(t)= & \frac{(a+b r)}{L} \sum_{k=1}^{L}\left[\sigma_{k}(t)+h\left(\rho^{(k)}(t)-1 / 2\right)\right] \\
& -\left[\sigma_{i}(t)+h\left(\rho^{(k)}(t)-1 / 2\right)\right]
\end{aligned}
$$

where

$$
\rho^{(k)}(t)=\frac{1}{\sum_{j=1}^{L} A_{k j}} \sum_{j=1}^{L} \sigma_{j}(t) A_{k j}
$$

Here the formula are defined for any directed graph. $A_{i j}$, $i, j=1, \ldots, L$ is the adjacency matrix of the graph and we assume that there is only one player in each node. The return is 
governed by two parameters: one is deterministic and is denoted by $a$ and the other is non-deterministic, which stands for the noise which amplitude is $b$ since $r$ is an uniform random variable distributed in $[-1,1]$. Each player is characterized by its motivation level, a variable initially defined as $\sigma_{i} \in\{0,1\}$ : the players are motivated or unmotivated.

Each player $k=1, \ldots, L$ invests a quantity $S_{k}=\sigma_{k}+h\left(\rho^{(k)}-\right.$ $1 / 2)$ where the investment is a function of the individual motivation $\sigma_{k}$ and the motivation of the neighborhood represented by the function $h\left(\rho^{(k)}-1 / 2\right)$. This neighborhood influence assumes the values 0,1 or 2 respectively if $\rho^{(k)}$ is smaller than $1 / 2$, exactly $1 / 2$ and higher than $1 / 2$, where the quantity $\rho^{(k)}$ corresponds to density of motivation of the neighborhood of the player $k$.

A first original contribution of this communication is to extend some of the explored results in other contributions by the same author of this manuscript and collaborators. In this paper, I obtained new results for the game in one dimensional lattices, small worlds and in the complete graph under mean field approximation. We have introduced a new alternative return function that considers a continuous contribution of the neighborhood. Basically, we replaced $h\left(\rho^{(k)}-1 / 2\right)$ by $\rho^{(k)}$ in 1:

$$
R_{i}^{C}(t)=\frac{(a+b r)}{L} \sum_{k=1}^{L}\left[\sigma_{k}(t)+\rho^{(k)}(t)\right]-\left[\sigma_{i}(t)+\rho^{(i)}(t)\right]
$$

More precisely we have studied the effects of this continuous influence in diagrams $a \times \rho_{0}$, when the noise is off $(b=0)$ for different networks. In these diagrams, for each pair $\left(a, \rho_{0}\right)$ is attributed a symbolic value corresponding to the kind of behavior of $\rho(t)$ for long times (for the symmetry of the problem just 3 possible behaviors are observed in the 2-state motivation model). In our model, the evolutionary aspect comes from the update of the motivation to be performed according to the return achieved by each player. The return function as exposed in the equation 3 is particularly important in small world networks (SW) because in this case the survival probability is strongly dependent of $p$ as we will see in the future sections.

We also explore in this contribution a dynamics for evolution of the density considering the update of motivation according to the return of neighbors prescribed by Boltzmann weights (Gibbs sampling dynamics). In this case we extended the granularity of motivation, i.e., $\sigma_{i} \in\{0,1, \ldots, q-1\}$. Plots of the density of motivation as a function of time for different values of noise and different values of $q$ were performed.

Our manuscript is organized as follows: in the section II we present a brief overview about the model. Section III exposes a novel dynamics based on Gibbs sampling dynamics. In the section IV we present the diagrams $a \times \rho_{0}$ that describe all possible behaviors of the density of motivation as function of time for all possible values of $a$ starting from a initial density $\rho_{0}$. We have built a program that discover all possible "patterns" for $\rho$ evolving along time. We compare the diagrams using the two return functions (equations 1 and 3 ) here proposed. In the section $\mathrm{V}$ we present the results for the model in two dimensions. And last but not least, we present some conclusions of our results in the section VI.

\section{THE MODEL AND PREVIOUS WORKS}

We consider $L$ agents in the context of public good game, where each agent can invest a particular quantity $S_{i}$. Agents invest cooperatively, i.e., the average profit of the group influences the investment motivation level of each agent, modelled by a binary variable $\sigma_{i} \in\{0,1\}\left(\sigma_{i}=1\right.$ means an agent is motivated while $\sigma_{i}=0$ means it is not). This abstraction aims at capturing issues such as insider information and economic prospects as perceived by agents.

We update the motivation at each time step by the profit rate $g_{k}(t)$ :

$$
\sigma_{k}(t+1)=\left\{\begin{array}{cc}
\frac{1}{2}\left(1+\frac{R_{k}(t)}{\left|R_{k}(t)\right|}\right) & \text { if } R_{k}(t) \neq 0 \\
0 & \text { otherwise }
\end{array}\right.
$$

This update is based on a simple principle: an agent's wealth relies on the wealth of the group. However, since agents are autonomous and there is room for cheating, we end up with two kinds of situations: one in which everyone is cooperative, and another where different types of individual behaviors are simulated.

Our previous work [7] has explored a particular one dimensional lattice (1DP) with periodic boundary conditions $\left(\sigma_{0}=\sigma_{L}\right)$. For this particular contribution, the investment of player $i$ depends only of the motivation of the player in the left $\left(\sigma_{i-1}\right)$ and its proper motivation $\left(\sigma_{i}\right)$ that are added, i.e., $S_{i}=\sigma_{i}+\sigma_{i-1}$ which assumes values in $\{0,1,2\}$. In this case, we concluded (see [7]) that this dynamical system can be seen as a kind of cellular automata (CA) and we proved that the long-time behavior for the density of motivation is an oscillation between two positive values $\left(\rho_{1} \rightarrow \rho_{2} \rightarrow \rho_{1} \rightarrow \rho_{2} \ldots\right)$ or the system goes to the fixed points $\rho \rightarrow 0$ or $\rho \rightarrow 1$.
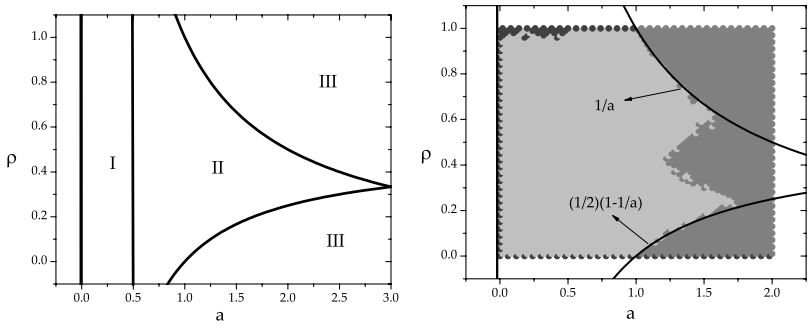

FIG. 1: Left plot: Phase diagram for the game in the case $b=0$ for 1DP (analytical results). Right plot: Computing simulations corresponding to $1 \mathrm{DP}$.

More precisely, the system is divided in three regions in the diagram $a \times \rho_{0}$, as can be seen in the Fig. 1: Region I represents the situation where the system is attracted to an oscillatory behavior, except for $\rho_{0}=1$ or 0 . In these two cases, the system is attracted to the fixed point $\rho=0$. In region II, the system is either attracted to $\rho=1$ or oscillates between 
two densities (no other situation can be observed). In region III, the system is always attracted to $\rho=1$. However, the analytical results are not precise enough to say where exactly in the region II the system goes to oscillatory case and where the system goes to 1 . In the next section, we will show simulations that explain this indetermination.

The same model was initially explored in small world networks [8] also just for the profit function of equation 1 . In this case, simulations were performed changing the influence of randomness of the small world $(p)$ on the probability of a particular player to reach the bankruptcy, i.e., different power laws are observed for this probability as a function of the time. Finally the case of complete graph (the player interacts with all other players in the limit $L \rightarrow \infty$ ) has been studied by the author and collaborators in other contribution [9]. The authors analytically proved that the density just can assume four values $\rho_{0}, 1-\rho_{0}, 0$ and 1 and for any region of diagram $(a, b)$, a four states Markov chain describes the equilibrium states for density of motivation.

In the next section we present a novel dynamics that will be applied for simulations of the model in two dimensions.

\section{A MODIFIED GIBBS SAMPLING FOR EVOLVING THE PUBLIC GOOD GAME ON GRAPHS}

In this part we propose a new dynamics for the public goods game to explore some other interesting evolutionary aspects alternatively to updating rules based on 4 . First of all we extended the possibilities of motivation, which now it is a $q$-state variable: $\sigma_{i}$ assumes values in $\{0,1, \ldots, q-1\}$.

An interesting evolutionary dynamics in the public good game can be simply based on the return of all neighbors. For updating its motivation the player copies the motivation of one of the neighbors giving higher probability as much higher as is its return. A natural choice is a sampling process known as Gibbs sampling or heath bath algorithm that prescribes a transition probability between two motivation level as:

$$
\operatorname{Pr}\left(\sigma_{i} \rightarrow \sigma_{j} ; t\right)=\frac{A_{i j} \exp \left[G_{j}(t) / T\right]}{\sum_{k=1}^{n} A_{i k} \exp \left[G_{k}(t) / T\right]} .
$$

where $G_{j}(t)$ is the return function (in this manuscript $R_{i}(t)$ or $\left.R_{i}^{C}(t)\right)$ and $T$ is an expansion parameter of the model that mimics the temperature in a magnetic model.

However, the use of these probabilities as so nullifies the effects of $a$ and $b$ since these probabilities are invariant over changes of $a$ and $b$ for both return functions used in this paper as can be easily seen. To solve this problem we added a extra rule in dynamics that considers only neighbors with positive return function for the sampling, reestablishing the dependency on parameters $a$ and $b$. In this case the formula 5 is rewritten as

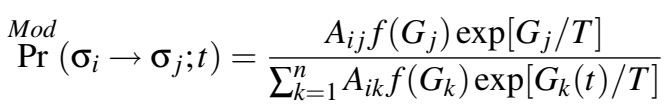

where $f\left(G_{j}\right)=1$ if $G_{j} \geq 0$ and $f\left(G_{j}\right)=0$ elsewhere.
In the section $\mathrm{V}$ we will show simulations of density of motivation based on the dynamics here described. We have studied the influence of noise, number of motivation levels $(q)$ and temperature in the public good game in two dimensions.

\section{RESULTS I: COMPARING PHASE DIAGRAMS UNDER DIFFERENT PROFIT FUNCTIONS}

We performed numerical simulations to build diagrams describing all possible evolutions starting from an initial configuration with density $\rho_{0}=1 / L \sum_{i=1}^{L} \sigma_{i}(0)$ for each parameter $a$ in absence of noise $(b=0)$. Here, it is considered a binary motivation level $\left(\sigma_{i} \in\{0,1\}\right)$ and for a suitable comparison, both return functions (see equations 1 and 3 ) were used.

In these conditions, the graphs studied in this manuscript presented 3 distinct behaviors: (1) the system goes to oscillatory behavior between two densities $\rho_{1}$ and $\rho_{2}$ or (2) all players become motivated $\rho \rightarrow 1$ or all players become unmotivated $\rho \rightarrow 0$.

A simplified version of the algorithm used to build these diagrams is shown in the table I. This algorithm attributes $\rho_{\infty}=0$ for the case which all players remain unmotivated at $t \rightarrow \infty, \rho_{\infty}=1$ when they remain motivated $t \rightarrow \infty$ and $\rho_{\infty}=1 / 2$ for oscillatory behavior at long times (here the attribution is symbolical). A threshold $n_{\text {rep }}$ is employed to recognize a "pattern" of $\rho_{\infty}=1$ (a robust sequence of 1's) because for the recognition of $\rho_{\infty}=0$ is necessary just to find the first occurrence $\rho=0$ and complementarily $\rho_{\infty}=1 / 2$ is assumed when none of the two first situations occur. In the Fig. 1 (right side) we show computing experiments that complete the lacks from theoretical predictions for 1DP (left side in the same figure). In the region II, we now can see clearly where is the region corresponding to $\rho=1$ and where the oscillatory behavior happens. The theoretical predictions [7] have claimed coexistence of these two behaviors in the region I, but it does not state anything about its partition. Clearly the most part is in oscillatory behavior since the dark gray corresponds to phase $\rho=0$, the light gray to the oscillatory behavior while the gray corresponds to phase $\rho=1$.
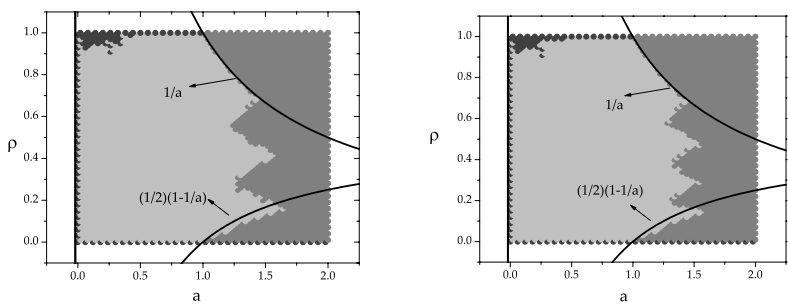

FIG. 2: Left plot: Diagram $a \times \rho_{0}$ for the $\rho(t \rightarrow \infty)$ in the onedimensional public good game using the return function from equation 1 Right plot: The same plot of left plot considering the alternative return function (eq.3).

We also study the one-dimensional lattice (under boundary periodic conditions) when each player interacts with its 


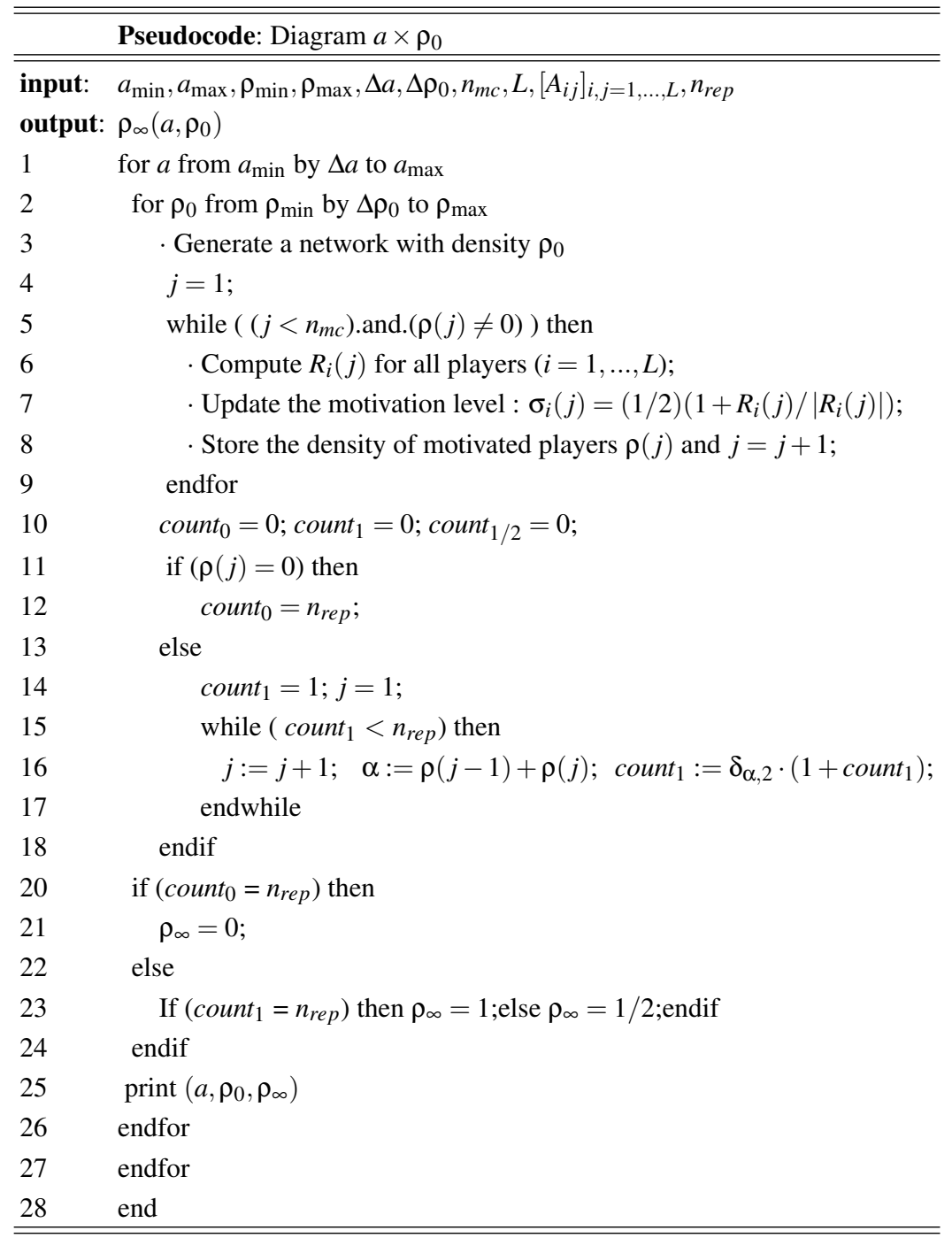

TABLE I: Algorithm

two nearest neighbors. We elaborate two diagrams considering for each one of them a different return function: $R_{i}(t)$ $\left(S_{i} \in\{0,1,2,3\}\right)$ and $R_{i}^{C}(t)\left(S_{i} \in\{0,1,3 / 2,2\}\right)$, respectively shown in the left and right side plots of the Fig. 2. In the left plot, we can note the arising of the one new "sawtooth" inside of oscillatory behavior region (region II from the Fig. 1). Surprisingly, when the return function $R_{i}^{C}(t)$ is used (right plot of 2), two smaller sawtooth appear.

The problem becomes more interesting if we consider time evolving in small world networks. For the sake of simplicity we consider in this paper small world networks generated from rings with nearest first and second neighbors $(k=2)$. First of all, we performed many simulations to test the influence of the different initial small worlds generated with same $p$ on the diagrams $a \times \rho_{0}$. Although we found the results to be highly sensible to different $p$ values, the diagrams $a \times \rho_{0}$ remain qualitatively unchangeable under different initial configurations of small worlds with the same $p$. After this check, we perform simulations changing the value of $p$. In the Fig. 3, we can see the different diagrams for different values of $p$. It was used $p=0,0.5$ and 1 for building the diagrams. The left plots were obtained using $R_{i}(t)$ and the right plots using the return function $R_{i}^{C}(t)$. It is particularly interesting the effects for the second return function.

For instance, if $p=0.5$, a lot of dark gray regions (no motivation regions) emerge in the diagram, differently from the plot using $R_{i}(t)$. This behavior motivated us to study the density of dark gray in an effective region of the diagram $a \times \rho_{0}$. More precisely we are interested in the complement of this amount, that in our problem can be interpreted as the survival probability of pro-social behavior:

$$
\operatorname{Pr}(\overline{\rho \rightarrow 0} \mid p)=1-\frac{1}{N} n(\rho \rightarrow 0 \mid p),
$$

where $n(\rho \rightarrow 0 \mid p)$ is the number of points $\left(a, \rho_{0}\right)$ such that $\rho \rightarrow 0$ given a small world with parameter $p$ and $N$ points on the grid $\left[a_{\min }, a_{\max }\right] \times\left[\rho_{\min }, \rho_{\max }\right]$. For our experiments, $\Delta a=0.025$ and $\Delta \rho_{0}=0.025$ were used as well as $a_{\min }=$ $\rho_{\min }=0$ and $a_{\max }=2$ and $\rho_{\max }=1$, resulting in $N=3200$ points. A plot of $\operatorname{Pr}(\rho \rightarrow 0 \mid p)$ as function of $p$ can be seen in 

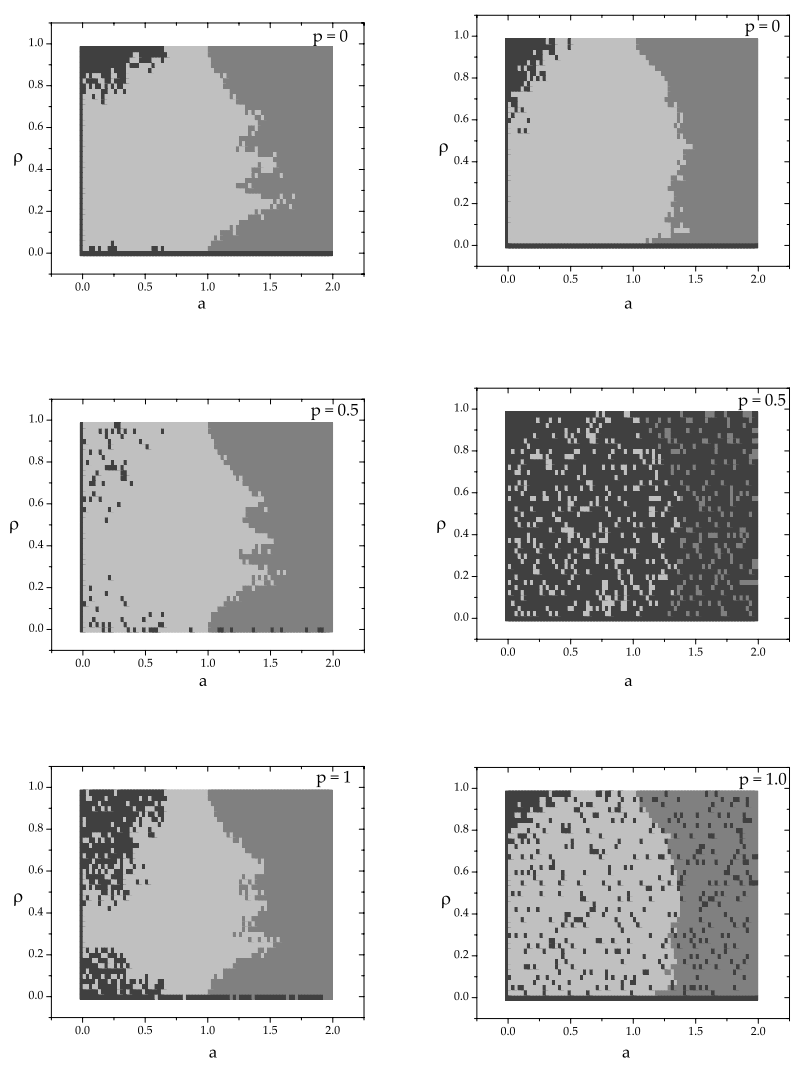

FIG. 3: The plots in the left side shows different diagrams for the different small worlds, respectively (from top to bottom) $p=0, p=$ 0.5 and $p=1$ using 1 . The right side plots corresponds to the same plots from return function $R_{i}^{C}(t)$.

the Fig. 4 (10 p values were simulated). A convex behavior as function of $p$ is observed for the probability of the population to remain motivated when $t \rightarrow \infty$. A minimum for this probability happens when $p \sim 0.6$, indicating less pro-social behavior in intermediate small worlds.

It is important to mention that we also simulated under the same conditions $b=0$ and using binary motivation level, the diagrams $a \times \rho_{0}$ for the model in two dimensions using lattices $50 \times 50$ and both return functions here defined. The results found were highly similar to the case of a small world when $p=0$ (see again the two plots on the top of the Fig. 3) and for this reason were not shown in this paper.

We also explore some simulations for the case where a player interacts with all players in a complete graph $\left(A_{i j}=1\right.$ for all $i, j=1, \ldots, L)$ under mean field approximation $\rho(t)=$ $\lim _{L \rightarrow \infty}(1 / L) \sum_{i=1}^{L} \sigma_{i}(t)$. In this case the possibilities are reduced to four state of densities, since the population starts in $\rho(0)=\rho_{0}, \rho(t)$ just will assume values in $\left\{0, \rho_{0}, 1-\rho_{0}, 1\right\}$. No other possibility can occur because players in the same state can only change to the same state (for a extensive analysis of mean field approximation for this model, using the profit function 1 see [9]).

We have also compared the effects of two return functions

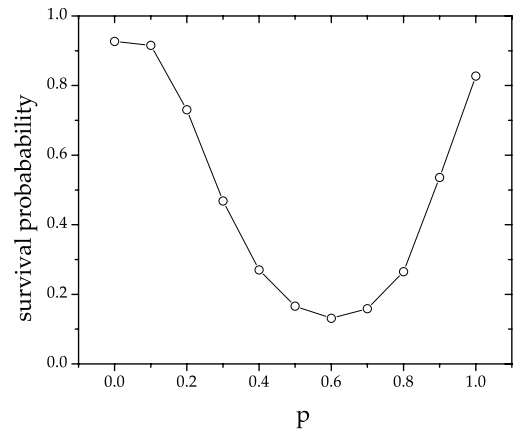

FIG. 4: Survival probability of motivation, $\operatorname{Pr}(\overline{\rho \rightarrow 0} \mid p)$ as function of disorder $p$ in the small world network.

for the complete graph under mean field behavior. For that, a graph with $L=10^{5}$ points was used. In the Fig. 5, the left side plot shows the diagram for return function $R_{i}(t)$. The straight lines comes from analytical results (see [9]) and are $\rho=7 / 2-(5 / 2) a, \rho=(5 / 2)(a-1), \rho=(15 / 4)(a-2 / 3)$ and $\rho=(7 / 2)-(15 / 4) a$. We can observe that our numerical simulations corroborate the analytical predictions. $\mathrm{Cu}-$ riously, when we use the return function from equation 3 the region corresponding to $\rho \rightarrow 0$ was reduced and the region corresponding to the oscillatory behavior enlarged. The region of oscillatory behavior before determined by straight lines $\rho=7 / 2-(5 / 2) a$ and $\rho=(5 / 2)(a-1)$ in the right is now determined by functions $\rho=a^{-1.75}$ and $\rho=1-a^{-1.75}$ respectively, which were numerically adjusted.

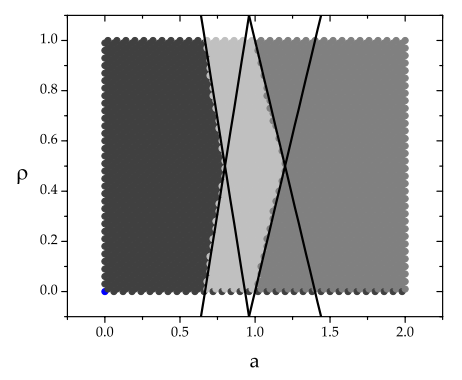

FIG. 5: Diagram $a \times \rho_{0}$ for the complete graph (mean field approximation). The left plot corresponds to the first return function and the right plot to second one.

In the next section we present results for the model in two dimensions. A novel dynamics developed in the section III is now applied for the game in two dimensions. A time-analysis of density of motivation was studied looking noise effects and motivation for more than two levels. 


\section{RESULTS II: MONTE CARLO SIMULATIONS FOR THE TWO DIMENSIONAL MODEL}

The noise effects $(b \neq 0)$ can highly influence the density of states in function of the time and performing simulations with a large number of repetitions is more interesting in this case. Looking for the model in two dimensions but still in two-level motivation, simulations were performed using only dynamics explored until now, which the updating is given according to the equation 4 . In this case only the return function $R_{i}^{C}(t)$ was used.

Considering a $n_{\text {sample }}=1000$ repetitions in a system $50 \times$ 50 , we analyzed time evolving of $\rho(t)$ considering $\left\langle\rho_{0}\right\rangle=1 / 2$. In the Fig. 6 are presented 4 plots, the three first corresponding to $b=0.15, b=0.30, b=0.50$ respectively and the last plot in this same figure shows two cases: $b=0.75$ and $b=0.90$. For all plots we consider $a=1$, which is exactly the border line between profit and financial damage.
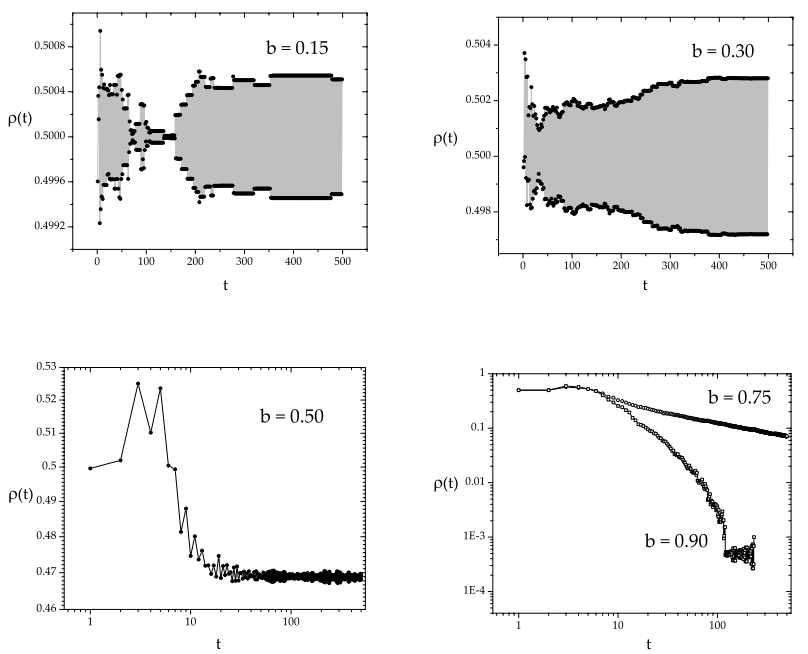

FIG. 6: Time evolution of density of motivation averaged over $n_{\text {sample }}=1000$ for different values of noise keeping $a=1$.

We can observe that lower values of noise $b=0.15$ and $b=0.30$ shows that in the most of repetitions the system goes to an oscillatory behavior when $t \rightarrow \infty\left(\rho_{1} \rightarrow \rho_{2} \rightarrow \rho_{1} \rightarrow \rho_{2} \ldots\right)$ with $\rho_{1}$ and $\rho_{2}$ being different for different runs. In some of the runs $\rho \rightarrow 0$ or $\rho \rightarrow 1$ can occurs, justifying the interesting behavior observed in the plot.

Finally for an intermediate value the model presents a saturation phenomena in the density of motivation as can be observed for $b=0.50$. For higher values of $b$ we finally arrive on a power law behavior when $b=0.75, \rho(t) \sim t^{-\theta}$ where $\theta=0.335(3)$. After setting $b=0.90$ an exponential tail is observed.

Here we test the approach developed in the section III for the two dimensional public good game. First of all we test the modified Gibbs sampling procedure fixing $T=10^{3}(\beta=$ $10^{-3}$ ) for the same $n_{\text {sample }}=1000$, but now using $a=2$ and $b=0$, changing the number of motivation levels $(q)^{1}$, as can be observed in the Fig. 7.

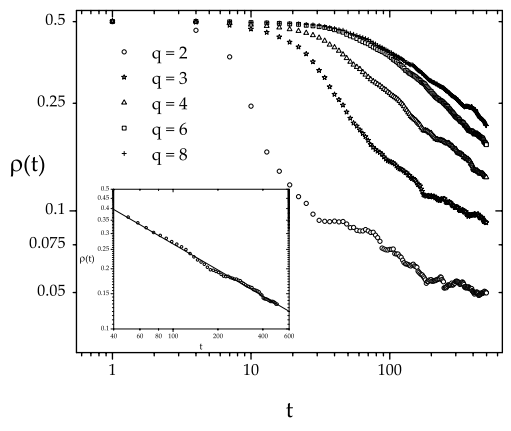

FIG. 7: Monte Carlo simulations of public good game in two dimensions using the modified Gibbs sampling. This figure shows the effects of the number of motivation states in time evolving of the density of motivation. For all cases, $a=2$ and $b=0$ were used .

The inside plot in this same figure shows particularly the case $q=3$, that notoriously present a power law behavior discarding the initial 50 MCsteps. We found $\theta=0.433(2)$.

Last but not least a complete analysis we study the noise and temperature effects on the two dimensional simulations using the modified gibbs sampling dynamics.
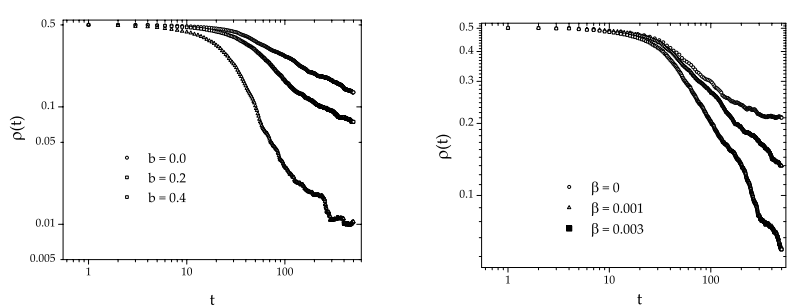

FIG. 8: This figure shows the noise effects (left plot) and temperature effects (right plot) in the density of motivated players using the Gibbs sampling dynamics.

Considering here also the parameters $a=2$ and $b=0$ we can see the noise effects (left side plot) and temperature effects (right side plot) in the Fig. 8. Both plots show that the system is highly sensible to these parameters, changing the density of motivation as a function of the time. In these plots we used $q=3$.

\section{CONCLUSIONS}

We have analyzed in this contribution a new way to explain how the pro-social behavior can emerge in an artificial soci-

\footnotetext{
${ }^{1}$ Here the density of motivation is normalized: $\rho(t)=(q L)^{-1} \sum_{i=1}^{L} \sigma_{i}(t)$
} 
ety based in a known game from experimental economics: the public good game. To model this behavior a motivation variable was introduced, based on previous contributions of the same author of this manuscript. This motivation initially imagined a binary variable which corresponds to the player being motivated or not, and is updated according to a return function. The investment in the public good is obtained according to combinations of motivations among neighbor players and an analysis of different graphs is performed. Our first contribution was to perform a comparative analysis of diagrams for the motivation average behavior at long times starting from a graph with different initial values of density of the motivated players, $\rho_{0}$, and different values of deterministic return parameter $a$. We introduced a new return function that directly considers the motivation average of the neighborhood. A comparison with the return function of previous works was performed. In small world networks our results shows the survival probability of the pro-social behavior has its lowest value in small worlds with $p \sim 0.6$.

Finally we have introduced a evolutionary dynamics to explore the game in the two dimensional lattice. In this case we analyzed the model considering the motivation with more possibilities $(q-$ states motivation). The motivation change of a player follows a modified Gibbs sampling prescription which establishes higher probability of copying the motivation of the neighbor with higher payoff - discarding the negative ones. Our results show a strong dependence of density of motivation with $q$ and $b$ (the noise amplitude of the return).

\section{Acknowledgments}

R. da Silva thank CNPq for the financial support to this work.
[1] G. Szäbó, G. Fáth, Evolutionary games on graphs, Phys. Rep. 446, 97 (2007).

[2] D. Ashlock, Evolutionary Computation for Modeling and Optimization, Springer, New York, (2005)

[3] J. M. Smith, Evolution and the Theory of Games, Cambridge University Press (1982)

[4] T. N. Cason, T. Saijo, and T. Yamato, Voluntary Participation and Spite in Public Good Provision Experiments: An International Comparison, Experimental Economics, 5, 133 (2002).

[5] O. Gurerk, B. Irlenbusch, and B. Rockenbach, Science, 312, 108 (2006).

[6] R. Mark Isaac, J. M. Walker, and A. Williams, Journal of Public
Economics 54, 1 (1994).

[7] R. da Silva, A. L. C. Bazzan, A. T. Baraviera, and S. R. Dahmen, Physica A, 371, 610 (2006).

[8] R. da Silva, A. T. Baraviera, S. R. Dahmen, and A. L. C. Bazzan, Dynamics of a Public Investiment Game: from NearestNeighbor Lattices to Small-World Network, Lecture Notes in Economic and Mathematical Systems 584, 221 (2006).

[9] R.da Silva, L. F. Guidi, and A. T. Baraviera, The public good game on complete graphs: Analytical and numerical results, preprint (2008) 